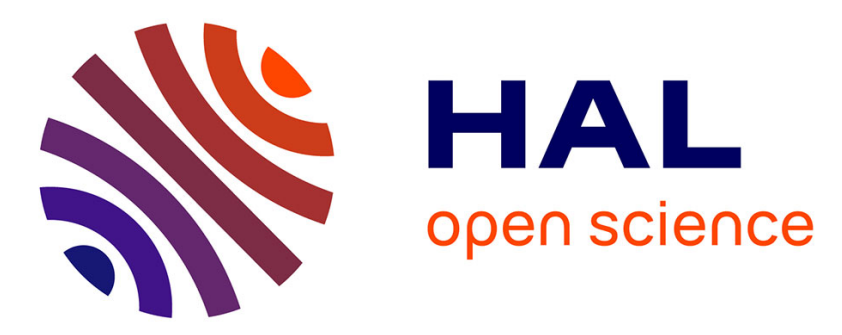

\title{
Electromagnetic study of the quality factor of pillar microcavities in the small diameter limit
}

Philippe Lalanne, Jean-Paul Hugonin, Jean-Michel Gérard

\section{To cite this version:}

Philippe Lalanne, Jean-Paul Hugonin, Jean-Michel Gérard. Electromagnetic study of the quality factor of pillar microcavities in the small diameter limit. Applied Physics Letters, 2004, 84 (23), pp.4726-4728. 10.1063/1.1759375 . hal-00872200

\section{HAL Id: hal-00872200 \\ https://hal-iogs.archives-ouvertes.fr/hal-00872200}

Submitted on 11 Oct 2013

HAL is a multi-disciplinary open access archive for the deposit and dissemination of scientific research documents, whether they are published or not. The documents may come from teaching and research institutions in France or abroad, or from public or private research centers.
L'archive ouverte pluridisciplinaire $\mathbf{H A L}$, est destinée au dépôt et à la diffusion de documents scientifiques de niveau recherche, publiés ou non, émanant des établissements d'enseignement et de recherche français ou étrangers, des laboratoires publics ou privés. 


\title{
Electromagnetic study of the quality factor of pillar microcavities in the small diameter limit
}

\author{
Ph. Lalanne ${ }^{\text {a) }}$ and J. P. Hugonin \\ Laboratoire Charles Fabry de l'Institut d'Optique, Centre National de la Recherche Scientifique, \\ 91403 Orsay Cedex, France \\ J. M. Gérard \\ CEA/DRFMC/SP2M, Nanophysics and Semiconductors Laboratory, 17, rue des Martyrs, \\ 38054 Grenoble Cedex, France
}

(Received 26 January 2004; accepted 7 April 2004; published online 20 May 2004)

\begin{abstract}
A thorough electromagnetic study of pillar microcavities reveals a surprising behavior for their $Q$ factors in the strong confinement limit. $Q$ displays a fast oscillatory variation as a function of the pillar diameter, and reaches values well in excess of the quality factor of the reference planar cavity. This behavior is explained by the hybrid character of the cavity mode in the small diameter limit, which results mainly from the electromagnetic coupling of the $\mathrm{HE}_{11}$ (i.e., fundamental) and $\mathrm{EH}_{11}$ guided modes of the cavity spacer through the associated Bloch modes in the Bragg mirrors.

(C) 2004 American Institute of Physics. [DOI: 10.1063/1.1759375]
\end{abstract}

Pillar microcavities have played a major role in the development of several optoelectronic devices, including vertical cavity surface-emitting lasers. ${ }^{1}$ Micropillars are also well suited to the realization of cavity quantum electrodynamic (CQED) experiments in the solid state, since they combine a small cavity volume $V$ and relatively high-quality factor $Q$. Clear demonstrations of the Purcell effect (spontaneous emission rate enhancement) have been obtained by incorporating InAs self-assembled quantum dots in pillar microcavities. ${ }^{2-4}$ The Purcell effect is the key for the efficient operation of the first single-mode solid-state single photon source, which implements a single quantum dot in a micropillar. ${ }^{3,4}$ In the small-diameter limit, other CQED effects such as the strong coupling regime or lasing for a single quantum dot might eventually be observed in the future, provided $Q$ remains large enough (i.e., $\mathrm{Q} \gg 1000$ typically). ${ }^{5}$ Experimentally, a strong decrease of $Q$ has been observed for submicron micropillars, even in the well studied GaAs/AlAs system; this behavior is commonly attributed to the detrimental effect of the scattering induced by the roughness of the etched sidewalls of the micropillars. ${ }^{6}$ In order to estimate the ultimate potential of micropillars for CQED and related applications, it therefore appears essential to study the intrinsic dependence of $Q$ versus the pillar diameter $d$. A good knowledge of the intrinsic cavity $Q$ is also important when one analyzes the performance of devices (such as lasers or single photon sources) ${ }^{7}$ in the presence of additional extrinsic losses.

Apart from Ref. 5 where micropillars are analyzed using finite-difference time-domain techniques for three pillar diameters $(d=0.3,0.4$, and $0.5 \mu \mathrm{m})$, previous studies rely on approximate methods, which assume that the field distribution can be written in a separable form as a function of the transverse and longitudinal coordinates. Such an approximation is not legitimate for small $d \mathrm{~s}$, and do not permit to study $Q$. In this letter, we present the results of an electromagnetic

${ }^{a)}$ Electronic mail: philippe.lalanne@ iota.u-psud.fr modelization of the $Q$ of pillar microcavities in the smalldiameter limit, which reveals amazing phenomena. $Q$ displays indeed a fast oscillatory variation as a function of the pillar diameter, and reaches values well in excess of the quality factor of the reference planar cavity. A simple approximate model is introduced to interpret the physical origin of this unexpected behavior.

Our calculation have been performed for the GaAs/AlAs system, which is both well mastered technologically and important for practical applications. In pillar microcavities, the photon confinement results from the combination of a waveguiding effect in the transverse direction and of a reflection by Bragg mirrors in the longitudinal direction. We consider here cylindrical micropillars having a circular cross section, with parameters typical of recently studied single photon sources. ${ }^{3}$ The micropillars are composed of a wavelength-thick GaAs cavity spacer, surrounded by a 9-period (top) and 25-period (bottom) distributed Bragg reflectors, see the bottom inset in Fig. 1. In the following, the reflectors are assumed to be composed of GaAs and AlAs layers, with refractive indices 3.495 and 2.94 at $\lambda_{0}=0.95$ $\mu \mathrm{m}$. Every layer of the reflector is assumed to have a quarterwave optical thickness for any diameter $d$ : For instance, all GaAs layers are $\lambda_{0} /\left(4 n_{1}\right)$ thick, $n_{1}$ being the effective index of the fundamental guided mode $\mathrm{HE}_{11}$ of a GaAs cylinder of diameter $d$. The thickness of the cavity layer $h$ is $\lambda_{0} / n_{1}$. This particular design rule ensures that the fundamental resonance wavelength of the micropillar remains very close to $\lambda_{0}$ (relative deviation lower than $10^{-3}$ ) over the entire range of diameters under study $\left(d>\lambda_{0} / n_{1}\right)$.

We have analyzed the electromagnetic properties of these micropillars by means of a frequency-domain Fouriermodal method. ${ }^{8}$ The method is rigorous; as one increases the number of Fourier harmonics retained for the computation, the calculated results tend toward the exact solution of Maxwell's equations. In brief, the method relies on a supercell technique which incorporates perfectly matched layers ${ }^{9}$ on the transversal side of the pillars. Since these layers absorb 


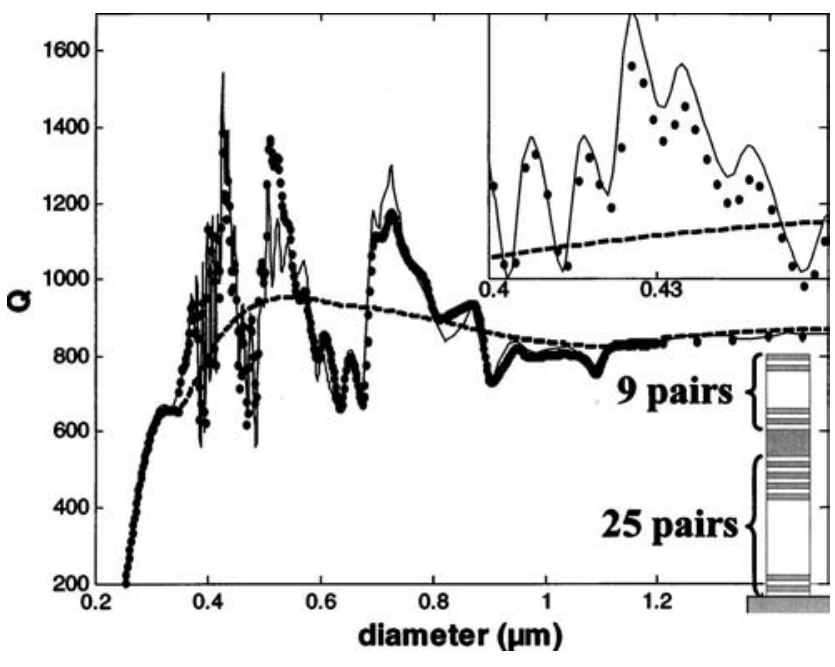

FIG. 1. $Q$ factor for pillar microcavities as a function of $d$. Solid curves are exact numerical data obtained with the Fourier modal method. The dotted (resp. dashed) curves represent the predictions obtained using the approximate model, and the expansion of the cavity mode on one (resp. two) guided modes in the spacer, and Bloch modes in the mirror. The top inset is an enlarged view for $d \approx 0.43 \mu \mathrm{m}$. Its vertical axis ranges from 730 to 1550 . The bottom inset is a sketch of the pillar geometry.

the evanescent and propagative radiation, the electromagnetic fields and their derivatives are null on every transversal boundaries of the supercell. Thus, they are periodic functions of the transverse coordinates and can be extended into Fourier series (plane-wave basis). This allows one to calculate the radiation and guided modes in a Fourier basis for each layer and to use a scattering matrix (S-matrix) approach to relate recursively the mode amplitudes in the different layers.

The solid curve in Fig. 1 shows the cavity $Q$ factors as a function of $d$. The $Q$ s are computed as $Q=\operatorname{Re}(\tilde{\lambda}) / 2 \operatorname{Im}(\tilde{\lambda})$, where $\tilde{\lambda}$ is the complex pole of the scattering matrix. Three different regimes are successively observed for decreasing $d \mathrm{~s}$ : For $d>1.2 \mu \mathrm{m}$ (which corresponds to $\approx 4 \lambda / n_{1}$ ), the calculated $Q$ s remain very similar to the planar cavity $Q(Q$ $=872$ ). When $1.2<d<0.35 \mu \mathrm{m}$, the $Q$ values surprisingly exhibit a complex oscillatory behavior. Finally, for $d<0.35$ $\mu \mathrm{m}$ (i.e., $\approx 1.3 \lambda / n_{1}$ ), $Q$ displays a fast decrease. In addition, it is noticeable that the oscillatory behavior presents a slow envelopelike variation and a fine structure, see the nearly sinusoidal pattern in the inset of Fig. 1. Since the effective cavity mode volume $V$ scales smoothly with $d$, the nontrivial oscillatory behavior is expected to reflect on the dynamical properties of an emitter inserted in the cavity. Computational results (not reported here due to limitations in space) for the enhancement of the total spontaneous-emission rate have indeed confirmed this statement. The oscillatory behavior has also been observed for microcavities with a 15 period top mirror. The $Q$ variation with $d$ shows again three different regimes with the same critical values of $d$, the same fine sinusoidal structure; only the slow envelopelike variation is shown to exhibit a faster oscillation behavior.

In order to analyze the physical origin of the cavity properties for small $d \mathrm{~s}$, we have developed an approximate modal method. We incorporate two main simplifications to the previous exact method. First, since the confinement resulting from the recursive mode energy exchange at all the interfaces of the Bragg reflectors is rather difficult to concep-

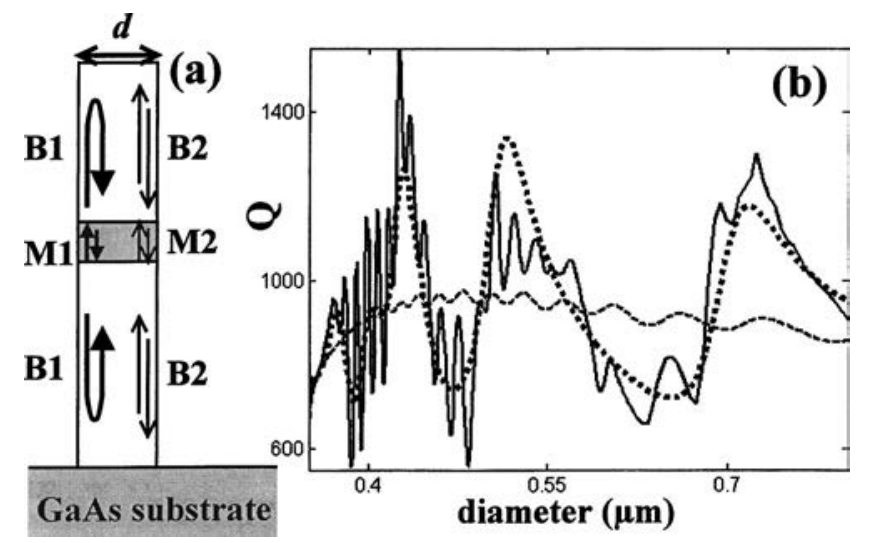

FIG. 2. (a) Schematic view of a pillar microcavity, and basis of spacer and mirror modes used to expand the cavity mode in the approximate method. (b) Role of $\mathrm{B}_{2}$ in the oscillatory behavior of $Q$ : The solid curve displays exact numerical data as in Fig. 1, whereas the dotted and dashed curves are obtained by removing the Bloch mode $\mathrm{B}_{2}$ from the decomposition basis for the top and bottom mirrors, respectively.

tualize, we introduce the Bloch modes of this periodic medium. Within this approach, the pillar geometry is simplified and is viewed as composed of three uniform sections, a GaAs spacer of height $h$ and two surrounding artificial uniform sections of heights 9 and 25 periods, see Fig. 2(a). Provided that the full continuum of Bloch modes is considered in the analysis, this first simplification does not introduce approximations since Maxwell's equations are still solved exactly. As we shall see, only two Bloch modes need to be considered to accurately predict the cavity $Q$ s. Second, we neglect radiation modes and only consider the guided modes for the modal expansion of the electromagnetic field inside the spacer. Finally, we calculate the coupling coefficient between modes at the spacer/mirror interfaces, ${ }^{10}$ and use a S-matrix formalism to estimate the resonant energy and $Q$ of the fundamental cavity mode.

The Bloch modes are computed as the eigenstates of the scattering matrix associated with one period of the Bragg mirrors. The computations are performed by expending the transverse fields in a plane-wave basis. ${ }^{8}$ For symmetry reasons, the Bloch modes can be labeled in a way similar to the guided modes of a dielectric waveguide. ${ }^{11}$ In the large diameter limit, the transverse dependence of the field is the same for the guided mode in the spacer and the corresponding Bloch mode of the Bragg mirror; coupling at the spacer/ mirror interface occurs only between modes with identical labelling. As a result, the fundamental cavity mode can be built using the fundamental guided mode $\mathrm{HE}_{11}$ in the spacer (hereafter labeled $\mathrm{M}_{1}$ for simplicity) and the $\mathrm{HE}_{11}$ Bloch mode in the mirror $\left(B_{1}\right)$. However, when the diameter is reduced, the overlap between these modes at the interface is no more perfect, so that they couple also with higher-order modes having the same symmetry. As shown now, the onset of these higher-order components in the expression of the fundamental cavity mode entails the complex dependence of $Q$ versus $d$.

The dashed curve in Fig. 1(a) represents the $Q$ factor calculated with the approximate modal method, when only $\mathrm{HE}_{11}$ modes are considered. $\mathrm{B}_{1}$ is a nonpropagating and nonleaky mode at the resonant wavelength $\lambda_{0}$ for all pillar diameters. Therefore, this situation corresponds to a standard 
Fabry-Perot model, relying on a cavity mode formed by the recirculation of the guided mode $\mathrm{M}_{1}$ of the spacer by the Bloch mode $\mathrm{B}_{1}$ of the top and bottom mirrors. The comparison to the exact numerical simulation shows that this model is accurate in the large diameter limit, as well as for $d<0.35$ $\mu \mathrm{m}$. In the latter case, all higher-order modes having the same symmetry as $\mathrm{HE}_{11}$ are leaky modes. The fast drop of $Q$ for decreasing $d \mathrm{~s}$ in this diameter range is due to the increasing impedance mismatch between $\mathrm{M}_{1}$ and $\mathrm{B}_{1}{ }^{12}$ and the associated radiation in free space. Note also that the approximate model predicts a slight increase of $Q$ for $d$ s going from 1.2 to $0.6 \mu \mathrm{m}$; this effect is due to the fact that the effective index contrast in the GaAs/AlAs Bragg reflector increases as a result for the lateral confinement.

In order to predict the oscillatory behavior for $Q$, it is however necessary to go beyond the Fabry-Perot model. For that purpose, we include now in the model the first higherorder modes having the same symmetry as $\mathrm{HE}_{11}$, i.e., the $\mathrm{EH}_{11}$ guided mode of the spacer $\left(\mathrm{M}_{2}\right)$ and the $\mathrm{EH}_{11}$ Bloch mode of the mirror $\left(\mathrm{B}_{2}\right)$. The corresponding $Q$ values are represented by the dots in Fig. 1. It is clear that this simple two-mode model reproduces fairly well the results of the exact numerical calculation. The physical origin of the oscillatory behavior of $Q$ can thus be understood by considering the properties of the Bloch mode $\mathrm{B}_{2}$. For $d>1.2 \mu \mathrm{m}, \mathrm{B}_{2}$ is-as $B_{1}$-a guided and nonpropagating Bloch mode of the mirror at the wavelength $\lambda_{0}$. As a result of the lateral confinement, the effective indices of the guided modes in the GaAs and AlAs layers, as well as the stop band of the mirrors, depend on $d$. Whereas the stopband for $\mathrm{HE}_{11}$ remains-by construction-centered on $\lambda_{0}$, it is shifted toward shorter wavelengths for $\mathrm{EH}_{11}$. For $0.35 \mu \mathrm{m}<d<1.2$ $\mu \mathrm{m}, \lambda_{0}$ is no more within the stop band for $\mathrm{EH}_{11}$, and $\mathrm{B}_{2}$ becomes a guided and propagating Bloch mode.

This peculiar property of $\mathrm{B}_{2}$ leads to an oscillatory behavior for several important microcavity parameters. Let us focus first on the crossed modal reflectivity $\mathrm{R}_{12}$, which describes the backward scattering into $\mathrm{M}_{2}$ for the incident mode $\mathrm{M}_{1}$, when propagating upward in the spacer and impinging onto the top mirror. This backscattering results from the combination of three different mechanisms: (1) Excitation of the evanescent $B_{1}$ mode, which is reflected back by the distributed Bragg reflector and scatters into the downward propagating mode $\mathrm{M}_{2}$ at the mirror-spacer interface, (2) excitation of mode $B_{1}$, which is scattered into $B_{2}$ at the top interface between the mirror and air, $\mathrm{B}_{2}$ propagates freely through the top mirror and couples efficiently into $\mathrm{M}_{2}$, and (3) direct excitation of mode $B_{2}$, which is reflected by the top interface and couples to $\mathrm{M}_{2}$ after propagation through the mirror. The propagation of $\mathrm{B}_{2}$ through the mirror induces delays (which depend on $d$ ) between these various contributions, and a beating effect on $\mathrm{R}_{12}$. Similar considerations apply obviously for the reflection on the bottom mirror; since it is much thicker than the top one, faster oscillations are expected in that case. Due to these fluctuations of the cross modal reflectivities on the top and bottom mirrors, the hybrid character of the cavity mode as well as $Q$ are expected to oscillate as a function of $d$.
To support this analysis, we consider two study cases. In the first case, we restrict our mode basis to $\mathrm{B}_{1}$ and $\mathrm{B}_{2}$ in the bottom mirror, $M_{1}$ and $M_{2}$ in the spacer, and only mode $B_{1}$ in the top mirror, in order to neglect the coupling to $\mathrm{B}_{2}$ in the top mirror. The calculated $Q$ s are shown as the dashed curve in Fig. 2(b). They well reproduce the fine sinusoidal structure, but do not reflect the slow envelopelike variation. In the second study case, the coupling to $\mathrm{B}_{2}$ is neglected for the bottom mirror only. This time, the calculated $Q$ s [dotted curve in Fig. 2(b)] well reproduce the slow envelopelike character, but not the fine sinusoidal structure. This analysis evidences the key role played by the propagating Bloch modes into the electromagnetic properties of micropillars with small $d \mathrm{~s}$ : The slow envelopelike variation of $Q$ is primarily due to the propagation of $B_{2}$ through the short top mirror, while the fine oscillations are associated to the lengthy bottom mirror. For $d \gg \lambda_{0} / n$, the oscillation vanishes. Not only $\mathrm{B}_{2}$ becomes nonpropagative, but also the hybrid character of the cavity mode disappears in this "large diameter" limit, as mentionned previously.

To conclude, the quality factor $Q$ of submicron diameter GaAs/AlAs pillar microcavities displays a strong and fast dependence as a function of the pillar diameter. This effect is related to the hybrid nature of their fundamental mode, resulting from the coupling of $\mathrm{HE}_{11}$ and $\mathrm{EH}_{11}$ modes by reflection at the spacer/mirror interfaces, and to the propagative nature of the $\mathrm{EH}_{11}$ Bloch modes of the Bragg mirrors. These effects must be taken into account when designing micropillar for advanced optoelectronic applications, such as single photon sources. On a practical side, our results also show that approximate models based on the expansion of the resonant cavity mode on a small number (here 2) of guided modes (in the spacer) and Bloch modes (in the mirror) provide a good insight on the electromagnetic properties of small diameter micropillars.

The authors gratefully acknowledge fruitful discussions with B. Gayral and P. Chavel.

${ }^{1}$ Spontaneous Emission and Laser Oscillation in Microcavities, edited by H. Yokoyama and K. Ujihara (CRC Press, Boca Raton, 1995).

${ }^{2}$ J. M. Gérard, B. Sermage, B. Gayral, B. Legrand, E. Costard, and V. Thierry-Mieg, Phys. Rev. Lett. 81, 1110 (1998).

${ }^{3}$ E. Moreau, I. Robert, J. M. Gérard, I. Abram, L. Manin, and V. ThierryMieg, Appl. Phys. Lett. 79, 2865 (2001).

${ }^{4}$ C. Santori, D. Fattal, J. Vuckovic, G. Solomon, and Y. Yamamoto, Nature (London) 419, 594 (2002).

${ }^{5}$ J. Vuckovic, M. Pelton, A. Scherer, and Y. Yamamoto, Phys. Rev. A 66, 023808 (2002).

${ }^{6}$ T. Rivera, J. P. Debray, B. Legrand, L. Manin, and J. L. Oudar, Appl. Phys. Lett. 74, 911 (1999).

${ }^{7}$ J. M. Gérard, B. Gayral, and E. Moreau, cond \mat quant \phys 0207115 (2002).

${ }^{8}$ P. Lalanne and E. Silberstein, Opt. Lett. 25, 1092 (2000); E. Silberstein, P. Lalanne, J. P. Hugonin, and Q. Cao, J. Opt. Soc. Am. A 18, 2865 (2001).

${ }^{9}$ J. P. Bérenger, J. Comput. Phys. 114, 185 (1994).

${ }^{10}$ P. Lalanne and J. P. Hugonin, IEEE J. Quantum Electron. 39, 1430 (2003).

${ }^{11}$ A. W. Snyder and J. D. Love, Optical Waveguide Theory (Chapman and Hall, New York, 1983).

${ }^{12}$ M. Palamaru and P. Lalanne, Appl. Phys. Lett. 78, 1466 (2001). 